\title{
An Helium calorimeter for Anti-Deuteron identification in cosmic rays
}

\author{
F. Nozzoli, ${ }^{a, *}$ F. Dimiccoli, ${ }^{a}$ R. luppa, ${ }^{a, b}$ E. Ricci ${ }^{a, b}$ and P. Zuccon ${ }^{a, b}$ \\ ${ }^{a}$ INFN-TIFPA Trento Institute for Fundamental Physics and Applications \\ via Sommarive 14, I-38123 Trento, Italy \\ ${ }^{b}$ Department of Physics, University of Trento, \\ via Sommarive 14, I-38123 Trento, Italy \\ E-mail: Francesco.Nozzoli@unitn.it
}

The observation of sub-GeV antideuteron in the cosmic ray flux could be a very strong signature of dark matter annihilation in our galaxy. The goal of the Anti Deuteron Helium Detector (ADHD) project is to study the signatures offered by an high pressure Helium calorimeter for the identification of antideuterons in space. The performance of an high pressure (200 bar) Helium scintillator prototype, capable of fast timing, will be shown.

$3^{\text {rth }}$ International Cosmic Ray Conference (ICRC 2021)

July 12th - 23rd, 2021

Online - Berlin, Germany

${ }^{*}$ Presenter 


\section{Introduction}

Cosmic Rays (CR) are important tools for the indirect search of dark matter (DM).

Well motivated theories beyond the Standard Model contain viable dark matter candidates which could lead to a significant enhancement of the antiparticle component in CR due to DM annihilation or decay [1]. Most of the DM annihilation channels can provide positrons $\left(\mathrm{e}^{+}\right)$or antiprotons $(\bar{p})$ in the Galaxy, however a substantial background flux of these antiparticles in the Milky Way is expected from secondary interactions of primary cosmic rays with the Inter-Stellar medium; moreover an additional background contribution for $\mathrm{e}^{+}$is also expected from nearby pulsars.

Conversely, in the case of antideuterons $(\bar{d})$, the secondary background is expected to be very low and in particular is kinematically suppressed at low energy (sub-GeV). Therefore, the observation of sub-GeV antideuteron in the cosmic ray flux could be a very strong signature of dark matter (DM) annihilation in our Galaxy; this leads to an high interest in the development of new detection techniques for low energy antideuterons. Goal of the Anti Deuteron Helium Detector (ADHD) project is to study the signatures offered by an helium target for the identification of $\overline{\mathrm{d}}$ in space.

Typical lifetime for stopping $\overline{\mathrm{d}}$ (like stopping $\overline{\mathrm{p}}$ ) in the matter is of the order of $\sim$ ps. However, since 1991, the existence of long-living $(\sim \mu \mathrm{s})$ metastable states for stopping $\overline{\mathrm{p}}$ in helium target was measured [2,3]. These metastable states in helium have also been measured for other heavy negative particles $[4,5]$, such as $\pi^{-}$and $\mathrm{K}^{-}$. The theoretical description of the effect [6-10] is predicting that the metastable state lifetimes increase as the reduced mass squared, i.e. a slightly larger delay is expected for $\overline{\mathrm{d}}$ capture in helium with respect to the measured $\bar{p}$ case. The antiprotonic-helium metastable states are well understood and their existence is already used for other fundamental physics measurements like the antiproton to electron mass ratio [11].

The phenomenology for the formation of metastable states in helium can be summarized following the scheme of Fig. 1.

\section{Antideuteron identification with the ADHD experiment.}

A possible configuration for an helium-based $\overline{\mathrm{d}}$ detector is depicted in Fig. 2. The response of a similar detector to $\overline{\mathrm{d}}, \overline{\mathrm{p}}$ and to the main cosmic ray components (p, He, C, $\mathrm{e}^{-}$) has been simulated with the Geant4 10.5 package [12]. The inner part is a $\sim 20 \mathrm{~kg}$ scintillating helium calorimeter (HeCal) where the 400 bar gas is filling a $300 \mathrm{~L}(\varnothing=90$ $\mathrm{cm})$ spherical thermoplastic vessel. Vessel wall thickness of $\sim 3 \mathrm{~cm}$ (vessel mass $\sim 100 \mathrm{~kg}$ ) ensures a burst pressure larger than 800 bar. A similar vessel is already considered for He transportation in space [13].

Helium gas is a fast UV scintillator, having a light yield similar to other fast plastic/liquid scintillators and capable of $\sim$ ns timing performances [14]. The HeCal is surrounded by three layers, made by $4 \mathrm{~mm}$ thick plastic scintillator bars, providing velocity measurement $(\beta)$ by Time of Flight (ToF) and charge measurement $(\mathrm{Z})$ by ionization energy loss measurement $(d E / d X)$. It is assumed that with current technology such a ToF 


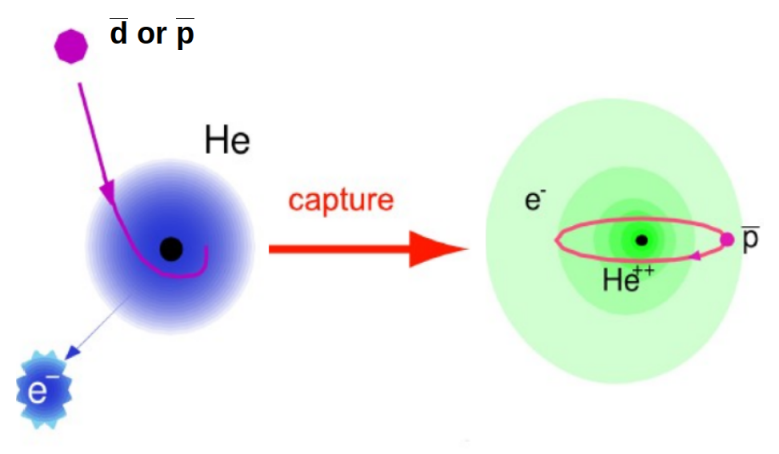

Figure 1: Stopping antiprotons and antideuterons (but also $\pi^{-}$and $\mathrm{K}^{-}$) can be captured by He and trapped in ( $\mu$ s living) metastable states before annihilation. Metastable states exists only for He target.

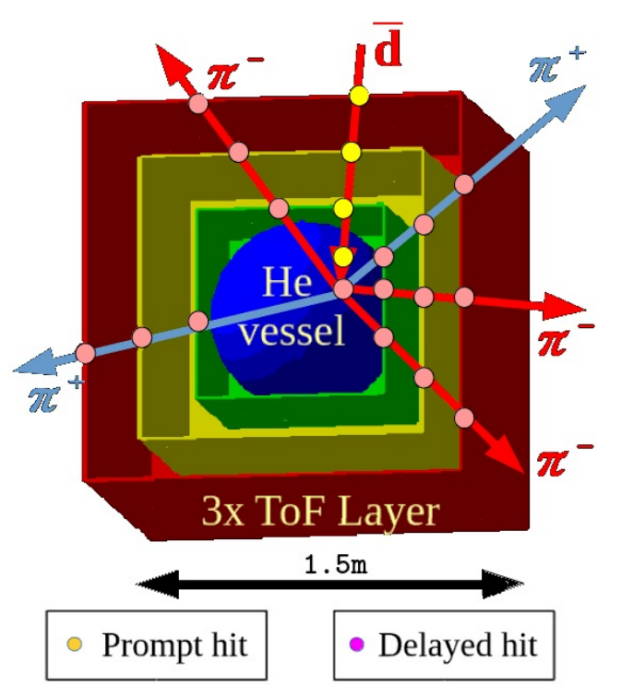

Figure 2: The ADHD detector is made by a 400 bar He gas calorimeter (HeCal) surrounded by three scintillator layers $(\mathrm{ToF}) . \overline{\mathrm{d}}$ are detected as a single in-going prompt track followed by few out-going delayed $\pi^{ \pm}$.

detector is capable of measuring $\beta$ with $5 \%$ resolution and energy loss with $10 \%$ resolution. This would imply time resolution of the order of $100 \mathrm{ps}$ and position resolution of the order of few $\mathrm{cm}$. Considering the energy loss in the ToF and in the vessel, a minimum kinetic energy of $\sim 60 \mathrm{MeV} / \mathrm{n}$ is necessary for $\overline{\mathrm{d}}$ to reach the He target. On the other hand, $\overline{\mathrm{d}}$ with kinetic energy larger than $140 \mathrm{MeV} / \mathrm{n}$ would typically cross the 400 bar He active region without stopping inside.

This defines the $60-140 \mathrm{MeV} / \mathrm{n}$ energy window explorable by ADHD in this configuration. Fig. 2 also shows the typical event topology for a stopping $\bar{d}$ within the He gas. The antiparticle initially produces three prompt hits (yellow) in the ToF and one prompt energy release (S1) in the HeCal; these prompt hits occurs within $10 \mathrm{~ns}$ (and are also produced by any other ionizing stopping particle). Then, only for $\overline{\mathrm{p}}$ and $\overline{\mathrm{d}}$, the antiparticle can be captured in the He metastable states and after a time delay going from several tens of ns to few $\mu$ s the annihilation occurs (pink delayed hits in Fig. 2). Typical $\pi^{ \pm}$multiplicity is 3.0 \pm 0.2 for each anti-nucleon annihilation at rest [15], therefore twice the number of delayed out-going tracks is expected for $\overline{\mathrm{d}}$ w.r.t. $\overline{\mathrm{p}}$. For the same reason, also the delayed signal (S2) in the HeCal for $\overline{\mathrm{d}}$ is expected to have a double amplitude w.r.t. the delayed signal for $\overline{\mathrm{p}}$. The characteristic temporal structure of S1/S2 signals as measured by HeCal for $\overline{\mathrm{d}}$ is shown in Fig. 3. The time gap from S1(prompt) to S2(delayed) is related to the metastability of He capture and is statistically distributed with $\tau_{c} \sim \mathrm{O}(1 \mu \mathrm{s})$. The $\mathrm{S} 1$ signal is related to the energy loss in the scintillating He, the amplitude measures the residual particle kinetic energy after the energy losses due to ToF and Vessel crossing. The duration of S1 signal is of the order of few ns that is the slowdown time for the particle in He. 


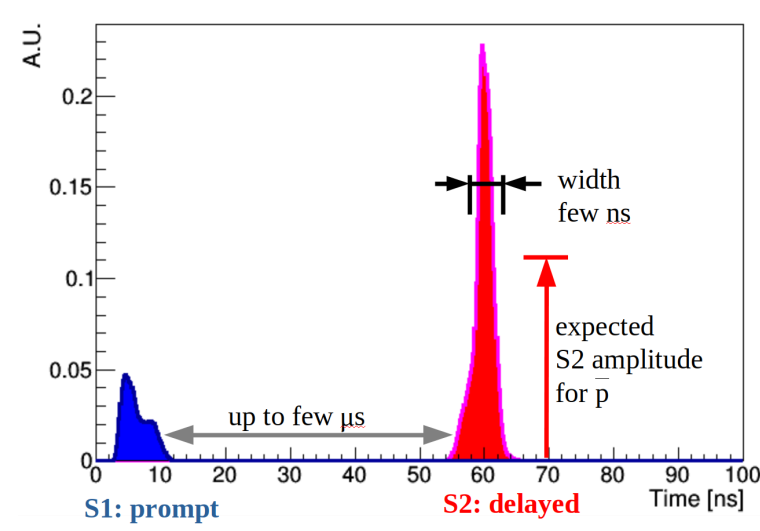

Figure 3: Typical HeCal signature for $\overline{\mathrm{d}}$ in ADHD detector.

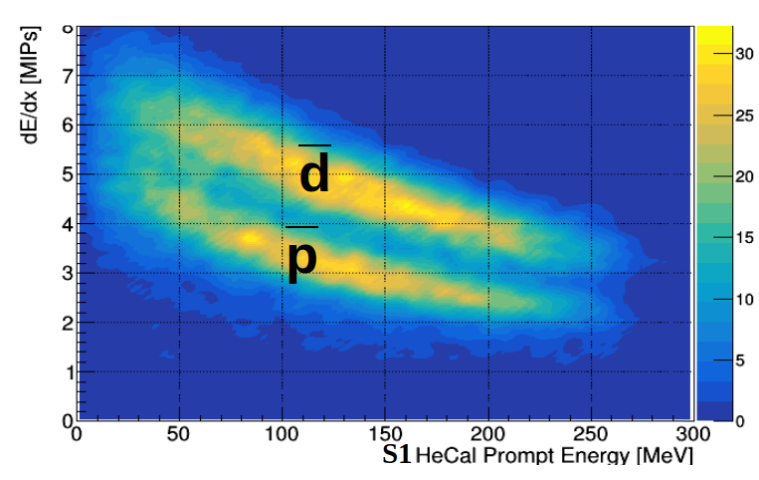

Figure 4: $\overline{\mathrm{p}}$ background is rejected thanks to the comparison of prompt track energy loss (and $\beta$ ) as measured by ToF with the total kinetic energy as measured by HeCal (S1).

The S2 signal is related to the number, velocity and trajectory of charged pions produced in the annihilation. The amplitude of S2 is not related to the particle kinetic energy, but measures the number of anti-nucleons. Typical S2 amplitude is higher than S1 and the duration of S2 signal is of the order of $\sim$ ns that is the transit time of pions to move from the helium to the vessel. This peculiar S1/S2 signature, together with the measurement of $\beta$ and $d E / d X$ (see fig. 4) for the in-going particle, is very distinctive for $\overline{\mathrm{d}}$ and $\overline{\mathrm{p}}$ acting as a powerful rejection for other $\mathrm{CR}$ components. Combining prompt and delayed event information, ADHD can identify a single $\overline{\mathrm{d}}$ over 1000 background $\overline{\mathrm{p}}$ in the $60-140 \mathrm{MeV} / \mathrm{n}$ range. Considering the expected $\overline{\mathrm{p}}$ flux, this technique would be able to test the presence of $\overline{\mathrm{d}}$ in cosmic rays down to a flux of $2-3 \times 10^{-6}\left(\mathrm{~m}^{2} \mathrm{~s} \text { sr } \mathrm{GeV} / \mathrm{n}\right)^{-1}$ with less than $1 \overline{\mathrm{p}}$ as background.

It is interesting to note that the ADHD expected sensitivity is similar to the ones expected with different techniques by other experiments in complementary energy windows.

\section{Measurements on HeCal prototype Arktis B-470.}

The timing and energy resolution of HeCal detector are key parameters for the ADHD project. Some measurements on a prototype based on the fast neutron detector B-470 Arktis Radiation Detectors [16] have been conducted in INFN-TIFPA laboratories in order to test the response of pressurized He gas as scintillator.

The Arktis B-470 detector consists of a stainless steel cylindrical chamber filled with pressurized ${ }^{4} \mathrm{He}$ gas close to 200 bar and two Hamamatsu-R580 photomultiplier tubes (PMTs) installed at the two ends of the chamber (see Fig. 5). The inner wall of the chamber is lined with a wavelength shifter to convert ultra-violet He scintillation to the optical wavelengths for the PMTs. Response of this detector to fast neutrons are extensively studied [14, 17-21]. However for the aim of ADHD project the response of He scintillation to charged (stopping) particles is necessary.

A preliminary test with muons from cosmic rays was performed. The B-470 detector was operated in coincidence with a $\left(10 \times 10 \times 30 \mathrm{~mm}^{3}\right)$ plastic scintillator and a small $(5 \times 5 \times 20$ 

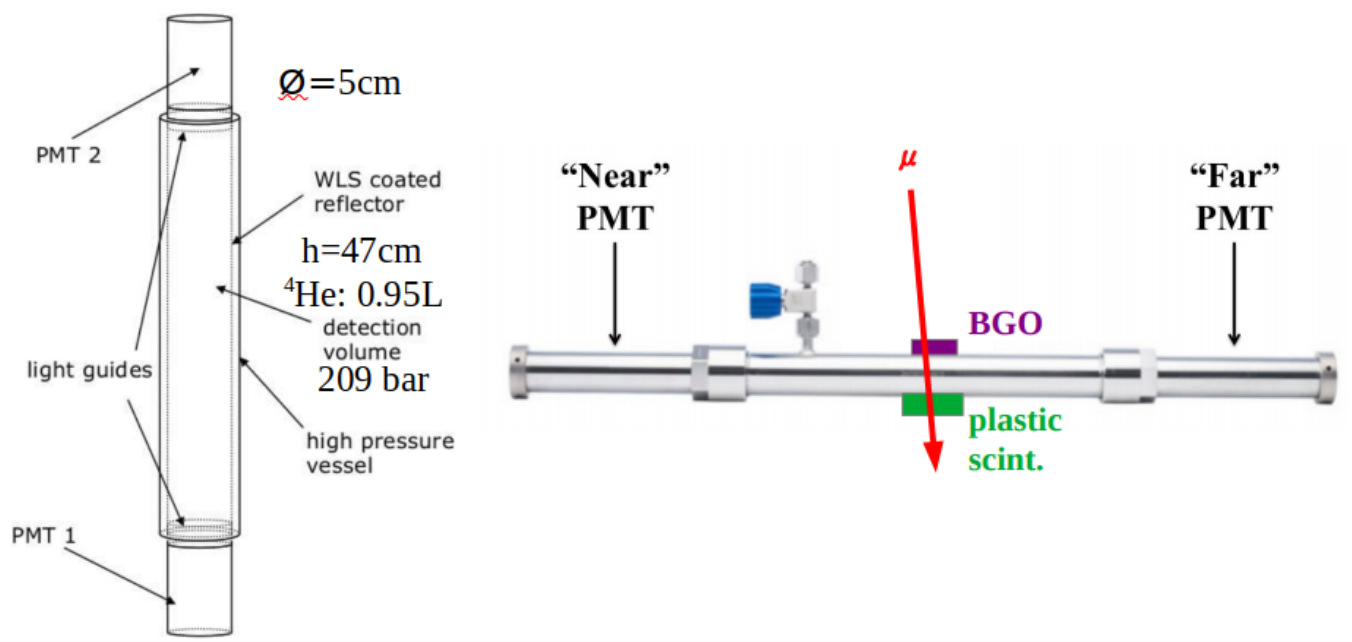

Figure 5: The Arktis B-470 detector used as a prototype of ADHD/HeCal.

$\mathrm{mm}^{3}$ ) BGO scintillator, placed in a telescopic configuration to detect crossing muons. The data was acquired with a LeCroy HDO9104-MS oscilloscope by sampling the detector waveforms at $20 \mathrm{Gs} / \mathrm{s}$. The "minimum" energy deposition in the helium volume was obtained for muon transversely crossing the detector diameter (depositing $\sim 0.3 \mathrm{MeV}$ in 200 bar helium), conversely the maximum energy deposition was obtained for muons crossing the whole detector (vertically placed, depositing $\sim 3 \mathrm{MeV}$ in 200 bar helium).

The time difference between the plastic scintillator trigger and a single side of the B-470 detector is shown in Fig. 6 and Fig. 7 for the two configurations, respectively.

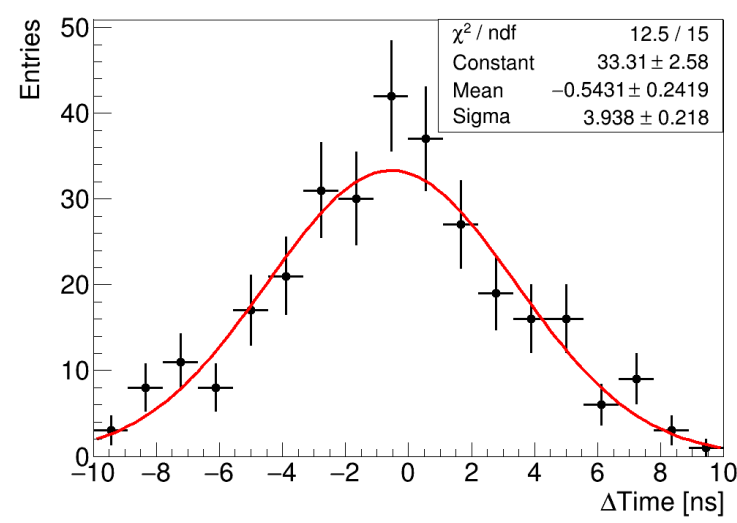

Figure 6: Time resolution of the Arktis B-470 detector measured with muons transversely crossing the detector $(\sim 300 \mathrm{keV}$ deposited).

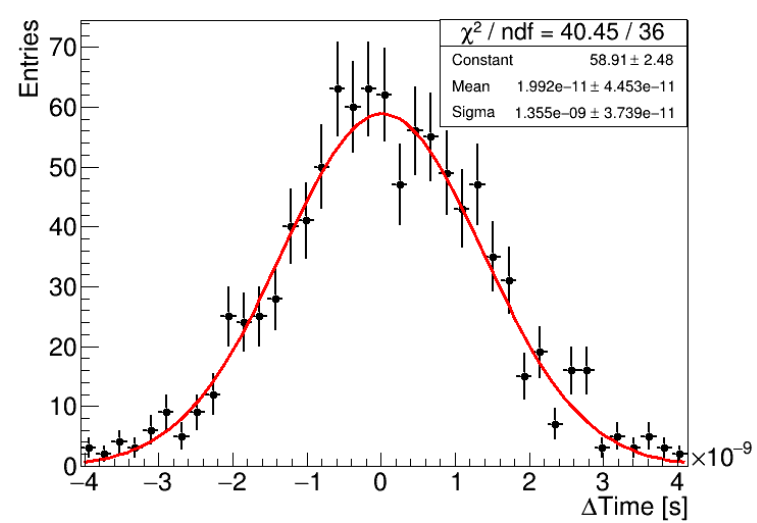

Figure 7: Time resolution of the Arktis B-470 detector measured with muons crossing the whole detector length $(\sim 3 \mathrm{MeV}$ deposited).

In particular the obtained (single side) time resolutions of $3.9 \pm 0.2 \mathrm{~ns}$ for $\sim 0.3 \mathrm{MeV}$ and $1.36 \pm 0.04 \mathrm{~ns}$ for $\sim 3 \mathrm{MeV}$ are in reasonable agreement with the time resolution of $2.4 \mathrm{~ns}$ achieved in [14] testing the same type of detector with a collimated ${ }^{137} \mathrm{Cs}$ source. This 

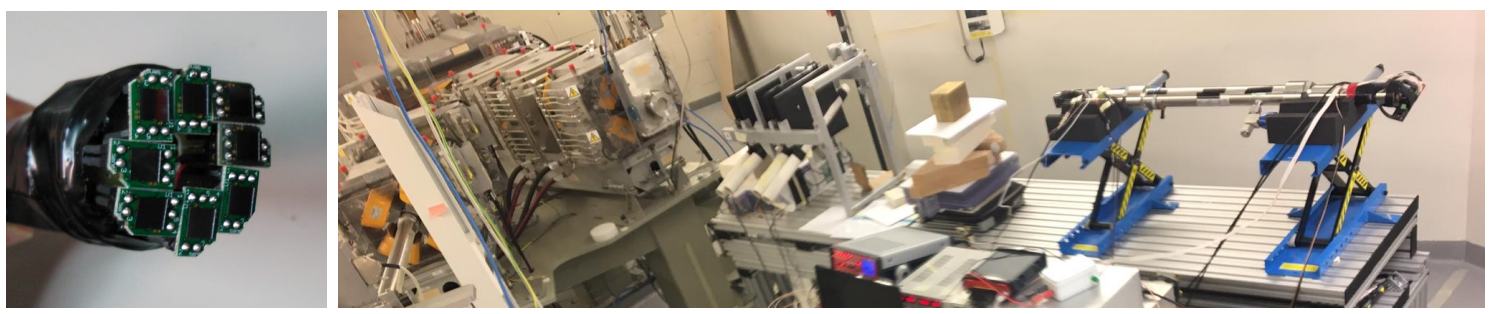

Figure 8: An array of 8x SiPM replaces one PMT of the Arktis B-470 detector (left picture). The detector prototype during the test at the Trento Proton Therapy facility (right picture).

preliminary measurement confirms the expected improvement of time resolution with the energy thanks to the incresing photon statistics, thus suggesting the capability to achieve a time resolution better than $1 / 2 \mathrm{~ns}$ for HeCal detecting $\bar{d}$ in the $60-140 \mathrm{MeV} / \mathrm{n}$ energy range.

\section{Perspectives and conclusions}

A test on the proton beam line in the experimental room of the Trento Proton Therapy Facility (Italy) [22] was pursued in the beginning of 2021. To allow the detection of the protons in the calorimeter avoiding PMT crossing, one PMT of the Arktis B-470 detector was replaced with an array of $8 \mathrm{x}$ Silicon Photo-Multipliers (see Fig. 8). The SiPM circular array is shielded by $20 \mathrm{~cm}$ of iron and a central hole of $1 \mathrm{~cm}$ allows the proton to enter in the Helium target by passing only through the optical window. The beam test was planned to characterize the B-470 detector with protons in the energy range $70-230 \mathrm{MeV}$ that is of interest for $\overline{\mathrm{d}}$ detection with ADHD. Analisys of data collected during the proton beam test is ongoing.

\subsection{Acknowledgments}

This work is funded by INFN grant $1959322 / 12 / 2017$.

\section{References}

[1] K. Hamaguchu et al., AMS-02 antiprotons from annihilating or decaying dark matter, Phys. Lett. B 747 (2015) 523.

[2] M. Iwasaki et al., Discovery of antiproton trapping by long-lived metastable states in liquid helium, Phys. Rev. Lett. 67 (1991) 1246.

[3] E. Widmann et al., Effects of impurity atoms and molecules on the lifetime of antiprotonic helium atoms, Phys. Rev. A 53 (1996) 3129.

[4] S.N. Nakamura et al., Negative-pion trapping by a metastable state in liquid helium, Phys. Rev.A 45 (1992) 6202.

[5] T. Yamazaki et al., Trapping of negative kaons by metastable states during the atomic cascade in liquid helium, Phys. Rev. Lett. 63 (1989) 1590. 
[6] G.T. Condo, On the absorption of negative pions by liquid helium, Phys. Lett. 9 (1964) 65.

[7] J.E. Russel, Metastable States of $\alpha \pi^{-} e^{-}, \alpha K^{-} e^{-}$and $\alpha \bar{p} e^{-}$Atoms, Phys. Rev. Lett. 23 (1969) 63.

[8] J.E. Russel, Interactions of an $\alpha K^{-} e^{-}$Atom with a He Atom, Phys. Rev. 188 (1969) 187.

[9] D.E. Wright and J.E. Russel, Energies of Highly Excited Helium-like Exotic Atoms, Phys. Rev. A 6 (1972) 2488.

[10] E. Widmann et al., Phase and density dependence of the delayed annihilation of metastable antiprotonic helium atoms in gas, liquid, and solid helium, Phys. Rev. A $51(1995) 2870$.

[11] M. Hori et al., Two-photon laser spectroscopy of antiprotonic helium and the antiprotonto-electron mass ratio, Nature 475 (2011) 484.

[12] J. Agostinelli et al., Geant4-a simulation toolkit, Nucl. Instrum. Methods A 506 (2003) 250.

[13] F. Benedic et al., Helium High Pressure Tanks at EADS Space Transportation New Technology with Thermoplastic Liner, Doc ID: ADA445482: EADS Report (2005).

[14] G. Davatz et al., Active Inspection of Nuclear Materials Using ${ }^{4} H e$ Scintillation Detectors, AIP Conf. Proc. 1412 (2011) 343.

[15] C. Amsler Proton-antiproton annihilation and meson spectroscopy with the Crystal Barrel, Rev. Mod. Phys. 70 (1998) 1239.

[16] Arktis Radiation Detectors Limited, 8045 Zurich, Switzerland.

[17] J.M. Lewis et al., Fission signal detection using helium-4 gas fast neutron scintillation detectors, Appl. Phys. Lett. 105 (2014) 014102.

[18] R.P. Kelley et al., Analysis of the scintillation mechanism in a pressurized ${ }^{4}$ He fast neutron detector using pulse shape fitting, AIP Adv. 5 (2015) 037144.

[19] R.P. Kelley et al., Neutron response function characterization of ${ }^{4}$ He scintillation detectors, hrefhttps://doi.org/10.1016/j.nima.2015.04.011 Nucl. Instrum. Methods A 793 (2015) 101.

[20] R. Jebali et al., A first comparison of the responses of a He-4-based fast-neutron detector and a NE-213 liquid-scintillator reference detector, Nucl. Instrum. Methods A 794 (2015) 102.

[21] T. Zhu et al., Improved fission neutron energy discrimination with ${ }^{4}$ He detectors through pulse filtering, Nucl. Instrum. Methods A 848 (2017) 137. 
[22] F. Tommasino et al., Proton beam characterization in the experimental room of the Trento Proton Therapy facility, Nucl. Instrum. Methods A 869 (2017) 15. 